\title{
Concepts of vertical and horizontal integration as an approach to integrated curriculum
}

\author{
Shahid Hassan \\ Unit of Medical Education, Faculty of Medicine and Health Sciences, Universiti Putra Malaysia
}

$\begin{array}{ll}\text { ARTICLE INFO } \\ \text { Received } & : \text { 26/02/2013 } \\ \text { Accepted } & : 31 / 03 / 2013 \\ \text { Published } & : 01 / 12 / 2013\end{array}$

KEYWORD

Integrated curriculum

Vertical integration

Horizontal integration

(c) Medical Education Department, School of Medical Sciences, Universiti Sains Malaysia. All rights reserved.

CORRESPONDING AUTHOR: Professor Dr Shahid Hassan, Unit of Medical Education, Faculty of Medicine and Health Sciences, Universiti Putra Malaysia

The current trend of integrated approach in medical education is a radical move from traditional curriculum that has been practiced for many years. The two most commonly found curriculum paradigms are based on either disciplinary or unified organizational structures however, 'some shade of grey' might exist (1). Traditional curriculum marked by disciplinewise model has been teaching basic sciences and clinical disciplines with a discrete subjectoriented instructional approach. Contrary to this integrated curriculum de-emphasizes the compartmentalization of disciplines and believes in unified interdisciplinary teaching. The impetus for radical change in curriculum design in undergraduate medical education is attributed to the principles of adult learning based on the outcome objectives. The objective behind reforms in curriculum change was aimed to produce clinically competent doctors who are able to meet the community health-care need. In its current shape the integrated curriculum is more focused to reducing the overloaded curriculum to deliver knowledge sufficient to relevant authentic patient's management problem. However, retention of knowledge, an important aspect of constructivist theory of learning was also emphasized in changing trend of integrated learning program in the new curriculum to ensure the competency of graduating physicians.

Setting up core objectives in curriculum is only of value if it achieves the required outcome. Only a good learning framework can guarantee that. A teaching strategy therefore becomes the most important aspect of the curriculum. The current trend in medical education is focused on how adults learn. The principles of adult learning determine that the students learn best when the objectives and outcome are clearly defined. Today's students need a) autonomy of learning 
that allow them to learn at their own pace, b) experiential environment that encourages them to brainstorm with their prior knowledge, c) early clinical exposure with real patient that provides context for learning and d) collaborative small group learning that ensures their self-directed and continuing learning. Professionalism and attention to attitudinal objectives as soft skills are the other most important aspects in the changing trend in medical education to which very little attention has been paid in the past (2). However, the emphasis is on identification of core objectives focused on clinical competency that a doctor needs to acquire to perform as a competent physician. Key to this concept of core clinical knowledge in undergraduate medical education is the vertical and horizontal integration of basic sciences and its relationship to para-clinical and clinical disciplines in an outcome-based curriculum.

In conventional terms, the vertical and horizontal integration is about documentation of curriculum for its organization considering two important dimensions, the sequence and the scope of the content in a curriculum (3). Sequence is about the vertical dimension of medical science, from basic to advance level of its training in medical education, whereas horizontal dimension is about breaking down the departmental barriers to integrate the basic sciences discipline (anatomy, physiology and biochemistry) with para-clinical (pharmacology, pathology and forensic medicine) and clinical disciplines (surgery, medicine and allied disciplines). In clinical education what students learn in clerkship (history taking, physical examination and clinical reasoning) is applicable to all specialties almost alike. Hence it is logical to deliver the curriculum in undergraduate medical education with an approach that promotes holistic patient care rather than the knowledge and the skills acquired in basic sciences and clinical disciplines isolated from each other as in traditional curriculum.

Documenting the list of information as an intended outcome of one level of training to build another level of training is the sequencing of curriculum organization or the vertical dimension. The same outcome grouped around a theme or topic determines the scope of the curriculum, which refers to horizontal dimensions and is more important for implementation of an integrated curriculum. Vertical dimension as a sequence of organization is regarded as the integration of basic sciences with clinical disciplines in integrated curriculum in a clinical context. Compared to this, horizontal integration in an integrated curriculum can be defined as the integration of knowledge and skills between the clinical disciplines in a curriculum, which has emphasis on early clinical exposure of medical students in training and holistic patient care approach in learning.

In order to facilitate vertical and horizontal integration in the curriculum, an integrated learning program is essential in which students are taught through authentic clinical cases either as real or simulated/standardized patients. Alternatively, paper patients can also be created for problem based learning with intend of either problem analyzing or problem solving depending on students' level of training in preclinical and clinical years respectively. This essentially refers to a well-established approach grounded with a recognized teaching model called problem based learning (PBL), which is implemented either with intentions of problem analyzing in preclinical years or problem solving in clinical years (4). To achieve the outcome-based objectives of integrated curriculum it is very important to adopt the measurement tools used in assessment, which are consistent with the integrated learning program to assess the integrated pattern of knowledge and skills studied in curriculum (5).

Horizontal integration helps resolve concepts that arise in relation to interdisciplinary subjects. Vertical integration, however, allows the study of more complex cases, specifically based on the structured acquisition and application of concepts from the preceding horizontally integrated coursework in an integrated curriculum. The horizontal and vertical integration in a curriculum provides students with a learning environment, which is consistent with principles of adult learning and is explicitly 
student-centered, problem-based and selfdirected life-long learning. It promotes learning of basic science subjects integrated with clinical disciplines as early as preclinical years for betterunderstood pathophysiology and the underlying pathological phenomena in clinical education. The early exposure of students to clinical scenarios provides them with immediate opportunities to apply basic sciences knowledge relevant to real world with long-term retention and analytic clinical reasoning practiced in problem solving (6).

Sequence and scope in curriculum determine two different dimensions significant along a timeline. The events in curriculum can be described either occurring within the same timeframe or subsequent to one another. The former dimension, which refers to a particular topic, course or module, is a horizontal dimension called horizontal integration in integrated learning program. Delivering the curriculum with a particular learning method based on clinical problems (PBL) and developed as an organ or system of body organized in blocks or modules (cardiovascular, gastrointestinal or genitourinary) is the example of horizontal integration. This learning approach observes the integration of clinical disciplines currently organized to teach students in their preclinical phase in curriculum, which often is implemented with PBL methods in most of the medical schools. The latter integration, which refers to what follows a particular topic or course, is suppose to be the vertical dimension which in preclinical years of integrated learning program meets the criteria, both of integrating the basic and clinical sciences, as well as gradually building information from fundamental basic clinical knowledge to advance clinical knowledge. In the current curriculum in preclinical teaching commonly practiced in medical schools with PBL approach, knowledge is gradually developed from basic topics of cell and tissue, general pharmacology and general pathology in year one. Compared to this knowledge of complex anatomy, advance pharmacology and special pathology in year two is taught in an organ-system blocks that links the body structure and function to its sign and symptoms in clinical cases is an example of vertical integration in curriculum.

One might debate and question the integrated teaching and learning practiced in clinical years of curriculum in medical schools, which is often criticized for losing their grip on continuing basic sciences knowledge and pathological phenomena in clinical learning besides their inability to sequentially built on information gathered from the previous years to subsequent years. Students posted for clinical teaching in tertiary hospital, where mostly complex cases are referred for management, are simultaneously exposed to these cases in their scheduled posting of clinical phase in year 3, 4 and 5. It is not uncommon to see the practice of undergraduate curriculum with integrated learning and PBL approach in preclinical phase of years 1 and 2 suddenly changed to traditional learning in clinical years 3 and onwards. This denies the continuity of integrated curriculum through the entire 5 years program of undergraduate medical program in many medical schools in developing countries.

As a solution to this, a more comprehensive program with a gradual shift from basic clinical skills in clerkship learnt on less complex cases to advance clinical skills learnt on more complex cases should be introduced. However, to successfully achieve this definitive learning model like PBL in preclinical years we will have to create or adopt clinical education with intend of problem solving in clinical years. Such models must base on principles of experiential learning, which is authentic, contextualized and stimulating for prior knowledge and reflective to build new knowledge (7). Learning models focused around themes such as pain, abdomen, locomotors apparatus, cardiovascular and respiratory or psychomotor problems and mental health can be organized for learning in clinical years like organ-system block teaching in preclinical years. In early clinical phase of year 3 clinical teaching can be centered on chronic diseases like it is practiced with student and patient's encounter taking place with feedback from supervisor and a peer in a special studentcentered outpatient clinic at the Maastricht 
University Medical Center (8). Student and the patient encounter can take place with feedback from supervisor on simple cases revolved around one of those themes created for clinical teaching. This can lead to development of advance model in subsequent clinical learning of years 4 and 5 with more complex and advance cases workup. Such models can be developed or adopted either utilizing a clinicopathological conferencing (CPC) model or analytic clinical reasoning (ACR) learning concepts based on Barrow's hypothetico-deductive process and Kaissrer's hypothesis testing with thinking aloud approach practiced in Quebec Medical School in undergraduate medical education (9).

Learning with problem solving authentic models designed with PBL approach and provision of carefully and periodically chosen simple to advance cases involving complex patients will ensure the sequence or the vertical integration in organization of curriculum. On the other hand studying multidisciplinary problems with concomitant or secondary lesions in complex patients to ensure horizontal integration can achieve the scope of organizing the content list in curriculum. The ongoing reforms in PBL approach with intent of problem solving and authentic learning in curriculum allows introduction of new teaching and learning strategies developed into well-recognized models like PBL model, which is consistent with challenges of clinical education in clinical phase of undergraduate medical education.

In conclusion an undergraduate curriculum in medical education can most effectively be integrated vertically into 5 years in which each year program is build on information gathered from the previous year, reflecting on graduates' experience and level of training. Alternatively curriculum can be demarcated into preclinical and clinical phase of studies in which basic sciences subjects are vertically integrated with clinical disciplines throughout the five years program in general and 2 years preclinical program in particular. Horizontal integration can be sought to include organ-system-based interdisciplinary training in preclinical years utilizing well established PBL model and interdisciplinary training in medicine, surgery and allied disciplines in clinical years, adopting or exploring new learning models in PBL approach. Implementation of curriculum with a well-strategized learning and teaching models in preclinical and clinical years with vertical and horizontal integration must also ensure the similar pattern of assessment. An assessment program consistent with level of clinical education should consider as far as possible, simple or less complex patients involved in early clinical years to testing of integrated clinical knowledge and skills with more advance and complex cases in later clinical years.

Faculty development is an integral and fundamental component of human resource development in a medical school, which has consciously adopted and followed an innovative as well as a challenging undergraduate medical education programme. We need sustainable faculty development programme in order to sustain the momentum of innovation. To ensure that this programme achieves its objectives and makes a difference in the training of doctors it is imperative to achieve support and commitment at the highest level is guaranteed. Perhaps the following sentiment by Bergquist sums up the whole conceptual framework and ideal for effective faculty development: 'A comprehensive faculty development programme is certainly not a part-time responsibility for one faculty member or administrator, nor is it a programme that can be carried out exclusively with volunteer help.' 'A faculty development programme begun with serious intent must be adequately staffed by professionals, with additional support provided by other available campus resources, including both faculty and students.' (8)

\section{Reference}

1. Orkwis PD, Walker BK, Jeng SM, Khosla PK, Slater GL, Simitses GJ. Horizontal and vertical integration of design: An approach to curriculum. Int. J. Engng Ed. 1997; 13 (3): 220-226.

2. Cookson JB. What's happening in undergraduate medical education? Postgrad 
Med J 1999; 75: 385-386 doi:

10.1136/pgmj.75.885.385

3. Posner GJ. Curriculum organization: basic concepts in analyzing the curriculum. Publisher Jane E Karpacz, McGraw Hill Companies Inc. New York. $3^{\text {rd }}$ Edition, $p$ 127-159, 2003.

4. Norman GR. Problem solving skills, solving problems and problem based learning. Medical Education, 1988; 22: 279-286.

5. Des Marchais JE, Nu Viet Vu. Developing and evaluating the students assessment system in the preclinical problem based curriculum at Sherbrooke. Academic Medicine, 1996; 71: 274-283.

6. Hassan S. How to develop a core curriculum in clinical skills for undergraduate medical teaching in the school of medical sciences at Universiti Sains Malaysia? Malaysian Journal of Medical Sciences, 2007; 14 (2); 4-10.

7. Bethell S, Morgan K. Problem-based and experiential learning: Engaging students in an undergraduate physical education module. Journal of Hospitality, Leisure, Sport and Tourism Education, 2011; 10 (1): 128-134.

8. O'Neil, Metcalfe, David. The core content of the undergraduate curriculum in Manchester. Medical Education, 1999; 33 (2): 121-129.

9. Des Marchais JE, Nu Viet Vu. Learning to become physician at Sherbrooke: A full switch to a student-centered MD program. Network Publication, Maastricht, 1996, 79111. 